\title{
Surface Chemical Modification of Multiwalled Carbon Nanotubes by a Wet-Mechanochemical Reaction
}

\author{
Lifei Chen, Huaqing Xie, Yang Li, and Wei Yu \\ School of Urban Development and Environmental Engineering, Shanghai Second Polytechnic University, Shanghai 201209, China
}

Correspondence should be addressed to Huaqing Xie, hqxie@eed.sspu.cn

Received 3 June 2008; Revised 10 August 2008; Accepted 5 September 2008

Recommended by Justin Holmes

A wet-mechanochemical reaction for surface modification of multiwalled carbon nanotubes (CNTs) has been developed. In the treatment, CNTs reacted with potassium hydroxide in alcohol solvent at room temperature under mechanical milling. The results from Fourier transmission infrared spectroscopy demonstrate that the nonreactive surfaces of CNTs have been modified successfully by multiple hydroxyl groups. Functionalized carbon nanotubes exhibit higher zeta potential values indicating their high dispersibility in polar solvents. XRD studies corroborate that the surface functionalization does not affect the basic crystal domain size of CNTs. TEM observations reveal that CNTs have been cut into shorter ones by the milling. Shortened CNTs with functionalized surfaces show good dispersion properties.

Copyright (c) 2008 Lifei Chen et al. This is an open access article distributed under the Creative Commons Attribution License, which permits unrestricted use, distribution, and reproduction in any medium, provided the original work is properly cited.

\section{INTRODUCTION}

Carbon nanotubes (CNTs) possess unique electronic, chemi$\mathrm{cal}$, and mechanical properties that make them leading materials for a variety of applications [1-3]. While many potential applications often need them to be prior purified and functionalized, because the bundling nature of CNTs originating from huge van der Waals binding energy [4] makes them difficult to be soluble in solvents either aqueous or nonaqueous even with dispersing materials. The lack of solubility and the difficult manipulation of CNTs in solvents impose great limitations to the practical applications of CNTs. Therefore, surface functionalization of CNTs has become the subject of research in recent years in order to improve their compatibility with matrix. A variety of methods, including covalent or noncovalent functionalization, have been developed to achieve effective deaggregation and dispersion [5-9]. Covalent reactions, such as cycloadditions [10], and addition reactions with nitrenes and diazonium salts $[11,12]$ were employed by many researchers in functionalizing the CNTs. However, the covalent functionalization has been found to deteriorate the intrinsic properties of single-walled carbon nanotubes (SWNTs) $[13,14]$. Noncovalent approaches, that is, supramolecule formation using amphiphilic surfactants, have been proved to be capable of debundling SWNTs ropes and stabilizing individual tubes while maintaining the SWNT integrity and intrinsic properties $[15,16]$. However, the addition of surfactants may contaminate the matrix. A generally known method to functionalize CNTs is chemical oxidation under ultrasonication or reflux conditions using mixtures of strong acids [17-21]. But the acid treatment results in serious loss of the materials as well as deterioration of CNT qualities. This method also holds the disadvantage of the difficulty in large-scale production. Recently, direct introduction of reactive monomers on CNT surfaces by organic chemical reactions has been reported [22, 23]. Another reported method to functionalize SWNTs is solid-phase mechanochemical reaction in which potassium hydroxide $(\mathrm{KOH})$ reacted with SWNTs and hydroxyl groups were added directly onto the surfaces of SWNTs at room temperature [24]. However, when this technique was applied in treating multiwalled CNTs (MWNTs), the reactants (KOH and CNTs) agglomerated severely and could not mix equably.

In this study, we present a new way to modify the surfaces of pristine multiwalled carbon nanotubes (PCNTs) in a large-scale production with no damage of CNT sidewalls. We name this method as wet-mechanochemical reaction, in which PCNTs react with $\mathrm{KOH}$ in ethanol solvent at room temperature under mechanical milling. Compared with solid-phase mechanochemical reaction, the 
wet-mechanochemical reaction, in addition to involving highly reactive centers generated by the mechanical energy imparted to the reaction system [24], makes materials contact effectively. The effects of milling time on surface functionalization of MWNTs have been studied in detail. The treated CNTs (TCNTs) are analyzed by Fourier transmission infrared spectroscopy (FT-IR), thermal gravimetric ananlysis (TGA), zeta potential measurements, X-ray powder diffraction (XRD), and transmission electron microscope (TEM).

\section{EXPERIMENTAL}

MWNTs (purity: 95\%) were purchased from Chengdu Organic Chemicals Co., Ltd., Chinese Academy of Sciences, and were used as received without further purification. $\mathrm{KOH}$ is analytical reagent.

Typically, mixtures of $0.4 \mathrm{~g}$ PCNTs and $8 \mathrm{~g} \mathrm{KOH}$, and ethanol were milled intensively using a ball-milling machine. Chemical reaction and mechanical action took place during the milling procedure. The mass ratio of agate balls to total reactants was $5: 1$. The treated time were selected as 4 hours, 15 hours, and 30 hours. The obtained mixtures were dissolved in deionized water and precipitated by centrifuge (Auegra TM 64R Centrifuge, Beckman Coulter, Calif, USA). Precipitation was repeated until the water became neutral to ensure a complete removal of $\mathrm{KOH}$ residues and the samples were dried at $80^{\circ} \mathrm{C}$ for 20 hours. Pure and functionalized TCNTs were obtained.

FT-IR spectra of TCNTs were measured on a spectrophotometer (Vertex 70, Germany). TGA measurements were carried out on a series thermal analysis system (NETZSCH STA449C, Germany) under a flowing nitrogen atmosphere in the temperature range $50-900^{\circ} \mathrm{C}$ at a scan rate of $10^{\circ} \mathrm{C} / \mathrm{min}$. Zeta potentials of PCNTs and TCNTs were measured at a concentration of $0.01 \mathrm{wt} \%$ using a zetaplus analyzer (Nano ZS, Malvern company, UK) by electrophoresis light scattering method. PCNTs and TCNTs were dispersed in $10 \mathrm{mmol} / \mathrm{L}$ $\mathrm{NaCl}$ solutions by ultrasonication to ensure constant ionic strength, and the $\mathrm{pH}$ of the suspension was adjusted by adding either $\mathrm{HCl}$ or $\mathrm{NaOH}$. However, XRD measurements were recorded using an X-ray diffractometer (D8-Advance, Germany) equipped with a back monochromator operating at $40 \mathrm{kV}$, and a copper cathode was used as X-ray source $(\lambda=0.154 \mathrm{~nm})$. XRD patterns were recorded from 10 to $80^{\circ}(2 \theta)$ with a scanning step of $0.01^{\circ}$. Morphological characterization of carbon nanotubes was performed using transmission electron microscope (JEOL 2010, Japan).

\section{RESULTS AND DISCUSSION}

The nature of surface groups of PCNTs and TCNTs was studied by FT-IR and the results are shown in Figure 1. The FT-IR spectrum of TCNTs is obviously different from that of PCNTs. The detectable transmission band at $1230 \mathrm{~cm}^{-1}$ and $1574 \mathrm{~cm}^{-1}$ observed for the PCNTs in the studied wavenumber range (Figure 1(a)) are assigned to the carbon skeleton [25]. The transmission bands turned weak in the corresponding FT-IR spectrum (Figure 1(b)) for TCNTs. The signal at $1630 \mathrm{~cm}^{-1}$ in spectra of TCNTs and PCNTs

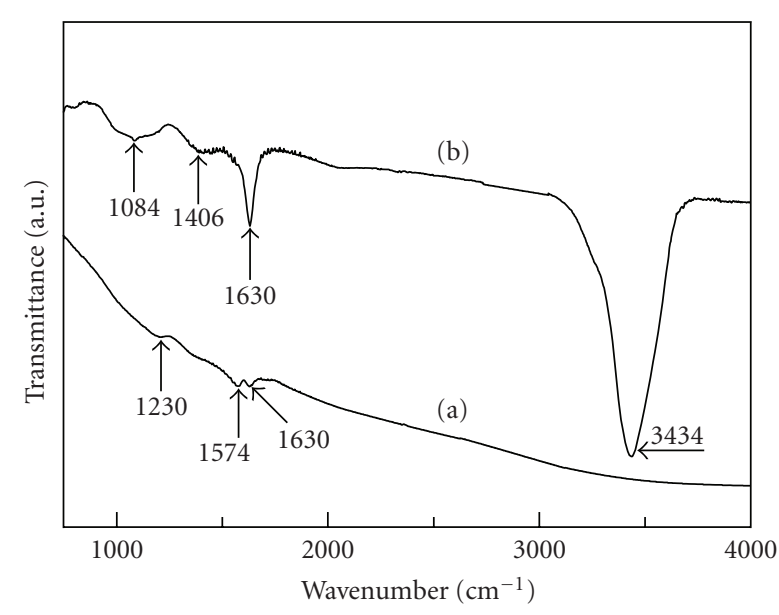

(a) PCNTs

(b) TCNTs

Figure 1: FT-IR spectra of (a) PCNTs and (b) TCNTs.

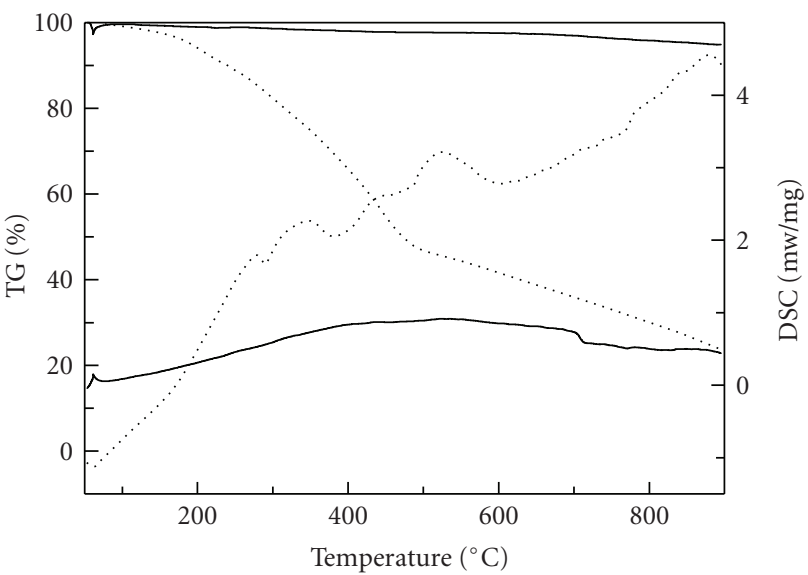

FIGURE 2: TG-DSC curves of PCNTs (solid line) and TCNTs (broken line).

is due to contaminating water [26]. The TCNTs presented extra peaks at $1084 \mathrm{~cm}^{-1}$, which can be assigned to C$\mathrm{O}-\mathrm{C}$ stretching group. The peak at $1406 \mathrm{~cm}^{-1}$ can be interpreted to the characteristic of hydroxyl bond $(\mathrm{O}-\mathrm{H})$. In addition, the very broad transmission band centered at $3434 \mathrm{~cm}^{-1}$ with high intensity of FTIR spectrum for TCNTs should be attributed to the hydrogen bond -OH. The FT-IR results obviously show that the hydroxyl groups have been introduced onto the surfaces of TCNTs. As a result, TCNTs can be dispersed in water and other polar solvent without surfactants.

Further evidence for functionalization of PCNTs by hydroxyl groups was provided by TGA. TGA provided useful information about functionalized CNTs because most functional moieties on CNTs are thermally unstable [27], that is, most of the organic functional groups are decomposed before the onset of CNTs weight loss [28, 29]. Figure 2 shows the thermograms of PCNTs and TCNTs in the range 40$900^{\circ} \mathrm{C}$. The weight loss also includes degassing and solvent 


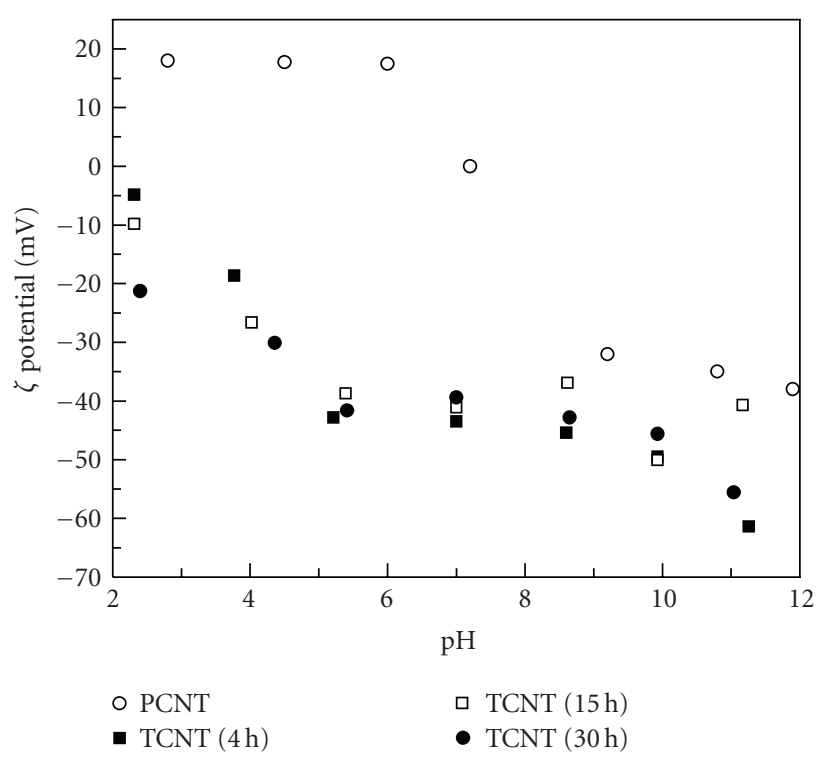

FIgUre 3: Zeta potential as a function of $\mathrm{pH}$ for PCNTs and TCNTs with different treated times.

evaporation at low temperatures [27]. The weight loss at $40-200^{\circ} \mathrm{C}$ accounted for approximately $10 \%$. The weight loss in the range of $200-480^{\circ} \mathrm{C}$ should be attributed to the decomposition between the functional groups and the CNTs. There is little weight loss of PCNTs as shown in Figure 2. The appearance of only two peaks at about 340 and $520^{\circ} \mathrm{C}$ in DSC curve also confirmed that there was little disordered carbon material in the TCNT sample, as the characteristic combustion temperature of disordered carbon usually emerges at around $400^{\circ} \mathrm{C}[30]$.

Zeta potentials as a function of $\mathrm{pH}$ for PCNT and TCNT suspension were determined and given in Figure 3. As seen in Figure 3(a), PCNTs have an isoelectric point (IEP) at $\mathrm{pH}$ 7.1. In TCNT suspensions, the IEP moves to a much acidic value at $\mathrm{pH} 3.7$ when the treated time was 4 hours. Zeta potential values of TCNT suspension were negative in all studied $\mathrm{pH}$ values and more negative with increase of treated time. This tendency was usually observed on ceramic suspensions mixed with anionic dispersants [31]. The changes in zeta potential values might be attributed to the surface chemical modification of CNTs, which introduces the hydroxyl groups onto the surfaces of CNTs. The result was consistent with that obtained from FT-IR. Furthermore, the more negative zeta potential values of TCNT dispersions indicate their considerable stabilities. Earlier reports on acid functionalized SWNTs reveal that the obtainment of stable dispersion of CNTs in water is due to the formation of electrical double layer in which ionic accumulation of few angstrom thicknesses prevents the particle aggregation and thereby stabilizes the suspension [32]. In the present case, the hydroxyl groups on the surfaces of TCNTs lead to the formation of electrical double layer around the TCNTs, preventing their aggregation. Also, the hydroxyl groups increase the affinity between TCNTs and water and thereby increase the stability of TCNTs in water.

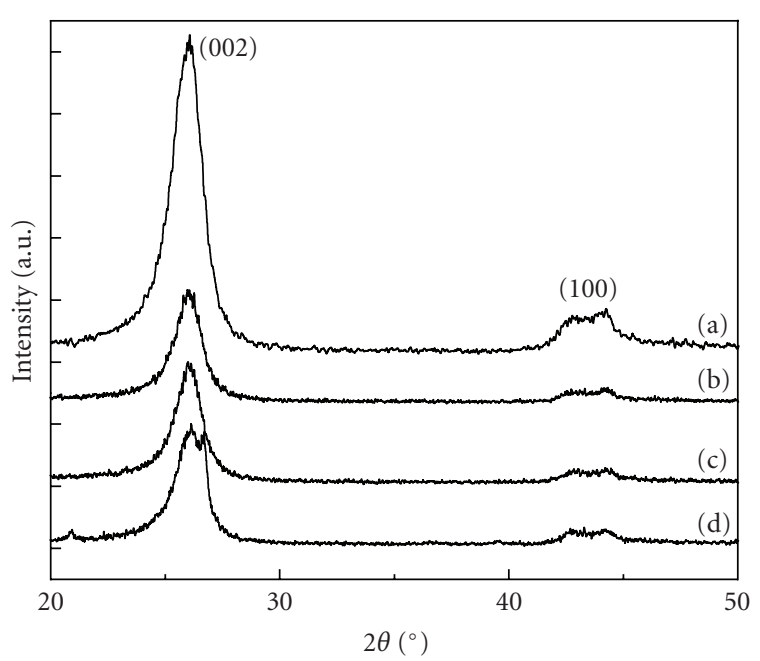
(a) PCNT
(c) $\operatorname{TCNT}(15 \mathrm{~h})$
(b) $\operatorname{TCNT}(4 \mathrm{~h})$
(d) $\operatorname{TCNT}(30 \mathrm{~h})$

FIGURE 4: XRD patterns of PCNTs and TCNTs with different treated times.

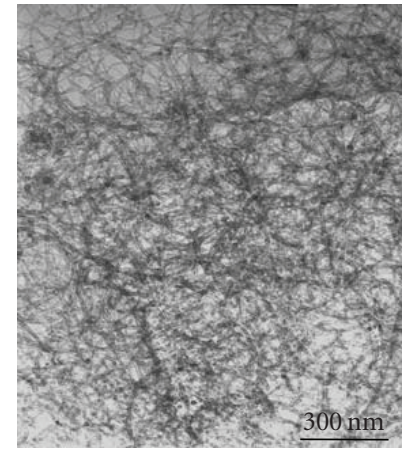

(a)

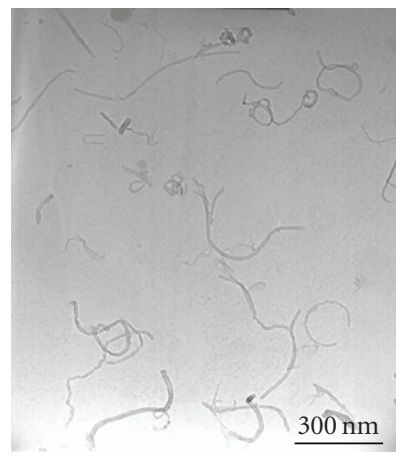

(b)
FIgure 5: TEM micrographs of (a) PCNTs and (b) TCNTs.

The XRD patterns of PCNTs and TCNTs with different treated times of 4 hours, 15 hours, and 30 hours are presented in Figure 4. The average interlayer spacing decided from (002) peak is $3.509 \AA$. The $(002)$ peak is broaden because of the finite number of layers and of the curvature of each tube. The broaden (10) bands indicate the curved sheet of the materials [33]. The average coherence length $\left(L_{\mathrm{c}}\right)$ of CNTs can be deduced from the width of the (002) Bragg peaks [34]. $L_{\mathrm{c}}$ of PCNTs and TCNTs with different treated times are $5.2 \mathrm{~nm}, 5.4 \mathrm{~nm}, 5.4 \mathrm{~nm}$, and $5.4 \mathrm{~nm}$, respectively. The values are very similar and show that the crystalline organization remains almost the same for all the samples. The results confirm that the wet-mechanochemical treatment does not damage the graphene layer organization. It indicates that the wet-mechanochemical reaction is feasible and facile to functionalize the surfaces of MWNTs. Furthermore, the intensity of (002) peak of PCNTs is much larger than TCNTs. This might be caused by the much lower packing density in 
TCNTs or some defects introduced in the process of wetmechanochemical treatment.

The morphological characterization was obtained using transmission electron microscope and the results are shown in Figure 5. PCNT and TCNT dispersions in ethanol dropped on copper grid covered with an amorphous carbon film, respectively. The samples are exposed to air at room temperature for drying until being suitable for the subsequent TEM analysis. PCNTs exhibit aggregation as observed by earlier researchers [35]. However, individual fibers are visible in the case of TCNTs. The debundling of the aggregates is attributed to the functionalization. Furthermore, TCNTs have been cut into short ones. It indicates that TCNTs with suitable length can be obtained by regulating the milling parameters.

\section{CONCLUSIONS}

Surface chemical functionalization of MWNTs was carried out by reaction of MWNTs with potassium hydroxide in ethanol solvent at room temperature under mechanical milling. FT-IR studies reveal that the hydroxyl groups have been introduced onto the surface of TCNTs. Thermogravimetric analysis of TCNTs exhibits degradation supporting the chemical functionalization. Higher zeta potential values for TCNTs indicate their excellent dispersing ability in solvents compared to PCNTs. Results of DSC and XRD show that the introduction of hydroxyl groups on TCNT surfaces has no effect on the crystallite species in the carbon nanotube bundles. TEM micrographs show that the carbon nanotubes have been cut during the mechanical milling. Preliminary studies on the influence of dispersion and aspect ratio of functionalized carbon nanotube reveal that it significantly affects the thermal conductivity of the nanofluids. The detailed study is underway in our laboratory and the results will be communicated shortly.

\section{ACKNOWLEDGMENTS}

This work was supported by the National High Technology Research and Development Program of China (2006AA05Z232), Shanghai Educational Development Foundation and Shanghai Municipal Education Commission (07SG56), and Innovation Program of Shanghai Municipal Education Commission (08ZZ99).

\section{REFERENCES}

[1] S. J. Tans, M. H. Devoret, H. Dai, et al., "Individual singlewall carbon nanotubes as quantum wires," Nature, vol. 386, no. 6624 , pp. 474-477, 1997.

[2] E. W. Wong, P. E. Sheehan, and C. M. Lieber, "Nanobeam mechanics: elasticity, strength, and toughness of nanorods and nanotubes," Science, vol. 277, no. 5334, pp. 1971-1975, 1997.

[3] M. Zhang, S. Fang, A. A. Zakhidov, et al., "Strong, transparent, multifunctional, carbon nanotube sheets," Science, vol. 309, no. 5738, pp. 1215-1219, 2005.

[4] A. Thess, R. Lee, P. Nikolaev, et al., "Crystalline ropes of metallic carbon nanotubes," Science, vol. 273, no. 5274, pp. 483-487, 1996.
[5] V. C. Moore, M. S. Strano, E. H. Haroz, et al., "Individually suspended single-walled carbon nanotubes in various surfactants," Nano Letters, vol. 3, no. 10, pp. 1379-1382, 2003.

[6] H. Wang, W. Zhou, D. L. Ho, et al., "Dispersing single-walled carbon nanotubes with surfactants: a small angle neutron scattering study," Nano Letters, vol. 4, no. 9, pp. 1789-1793, 2004.

[7] M. J. O'Connell, S. M. Bachilo, C. B. Huffman, et al., "Band gap fluorescence from individual single-walled carbon nanotubes," Science, vol. 297, no. 5581, pp. 593-596, 2002.

[8] J. L. Bahr, J. Yang, D. V. Kosynkin, M. J. Bronikowski, R. E. Smalley, and J. M. Tour, "Functionalization of carbon nanotubes by electrochemical reduction of aryl diazonium salts: a bucky paper electrode," Journal of the American Chemical Society, vol. 123, no. 27, pp. 6536-6542, 2001.

[9] A. Garg and S. B. Sinnott, "Effect of chemical functionalization on the mechanical properties of carbon nanotubes," Chemical Physics Letters, vol. 295, no. 4, pp. 273-278, 1998.

[10] V. Georgakilas, K. Kordatos, M. Prato, D. M. Guldi, M. Holzinger, and A. Hirsch, "Organic functionalization of carbon nanotubes," Journal of the American Chemical Society, vol. 124 , no. 5 , pp. 760-761, 2002.

[11] M. Holzinger, J. Steinmetz, D. Samaille, et al., "[2+1] cycloaddition for cross-linking SWCNTs," Carbon, vol. 42, no. 5-6, pp. 941-947, 2004.

[12] J. L. Bahr and J. M. Tour, "Highly functionalized carbon nanotubes using in situ generated diazonium compounds," Chemistry of Materials, vol. 13, no. 11, pp. 3823-3824, 2001.

[13] J. Chen, M. A. Hamon, H. Hu, et al., "Solution properties of single-walled carbon nanotubes," Science, vol. 282, no. 5386, pp. 95-98, 1998.

[14] Z. Yao, N. Braidy, G. A. Botton, and A. Adronov, "Polymerization from the surface of single-walled carbon nanotubespreparation and characterization of nanocomposites," Journal of the American Chemical Society, vol. 125, no. 51, pp. 1601516024, 2003.

[15] M. J. O'Connell, P. Boul, L. M. Ericson, et al., "Reversible water-solubilization of single-walled carbon nanotubes by polymer wrapping," Chemical Physics Letters, vol. 342, no. 3-4, pp. 265-271, 2001.

[16] R. J. Chen, Y. Zhan, D. Wang, and H. Dai, "Noncovalent sidewall functionalization of single-walled carbon nanotubes for protein immobilization," Journal of the American Chemical Society, vol. 123, no. 16, pp. 3838-3839, 2001.

[17] J. Liu, A. G. Rinzler, H. Dai, et al., "Fullerene pipes," Science, vol. 280, no. 5367, pp. 1253-1256, 1998.

[18] K. J. Ziegler, Z. Gu, H. Peng, E. L. Flor, R. H. Hauge, and R. E. Smalley, "Controlled oxidative cutting of single-walled carbon nanotubes," Journal of the American Chemical Society, vol. 127, no. 5, pp. 1541-1547, 2005.

[19] J. Zhang, H. Zou, Q. Qing, et al., "Effect of chemical oxidation on the structure of single-walled carbon nanotubes," Journal of Physical Chemistry B, vol. 107, no. 16, pp. 3712-3718, 2003.

[20] K. J. Ziegler, Z. Gu, J. Shaver, et al., "Cutting single-walled carbon nanotubes," Nanotechnology, vol. 16, no. 7, pp. S539S544, 2005.

[21] I. D. Rosca, F. Watari, M. Uo, and T. Akasaka, "Oxidation of multiwalled carbon nanotubes by nitric acid," Carbon, vol. 43, no. 15, pp. 3124-3131, 2005.

[22] C. A. Dyke and J. M. Tour, "Solvent-free functionalization of carbon nanotubes," Journal of the American Chemical Society, vol. 125, no. 5, pp. 1156-1157, 2003.

[23] K. S. Coleman, S. R. Bailey, S. Fogden, and M. L. H. Green, "Functionalization of single-walled carbon nanotubes via the 
Bingel reaction," Journal of the American Chemical Society, vol. 125, no. 29, pp. 8722-8723, 2003.

[24] H. Pan, L. Liu, Z.-X. Guo, et al., "Carbon nanotubols from mechanochemical reaction," Nano Letters, vol. 3, no. 1, pp. 2932, 2003.

[25] M. Liu, Y. Yang, T. Zhu, and Z. Liu, "Chemical modification of single-walled carbon nanotubes with peroxytrifluoroacetic acid," Carbon, vol. 43, no. 7, pp. 1470-1478, 2005.

[26] M. S. P. Shaffer, X. Fan, and A. H. Windle, "Dispersion and packing of carbon nanotubes," Carbon, vol. 36, no. 11, pp. 1603-1612, 1998.

[27] M. Liu, Y. Yang, T. Zhu, and Z. Liu, "Chemical modification of single-walled carbon nanotubes with peroxytrifluoroacetic acid," Carbon, vol. 43, no. 7, pp. 1470-1478, 2005.

[28] Y.-P. Sun, K. Fu, Y. Lin, and W. Huang, "Functionalized carbon nanotubes: properties and applications," Accounts of Chemical Research, vol. 35, no. 12, pp. 1096-1104, 2002.

[29] B. Z. Tang and H. Xu, "Preparation, alignment, and optical properties of soluble poly(phenylacetylene)-wrapped carbon nanotubes," Macromolecules, vol. 32, no. 8, pp. 2569-2576, 1999.

[30] L. Qingwen, Y. Hao, C. Yan, Z. Jin, and L. Zhongfan, "A scalable CVD synthesis of high-purity single-walled carbon nanotubes with porous $\mathrm{MgO}$ as support material," Journal of Materials Chemistry, vol. 12, no. 4, pp. 1179-1183, 2002.

[31] P. C. Hidber, T. J. Graule, and L. J. Gauckler, "Competitive adsorption of citric acid and poly(vinyl alcohol) onto alumina and its influence on the binder migration during drying," Journal of the American Ceramic Society, vol. 78, no. 7, pp. 1775-1780, 1995.

[32] H. Hu, A. Yu, E. Kim, et al., "Influence of the zeta potential on the dispersability and purification of single-walled carbon nanotubes," Journal of Physical Chemistry B, vol. 109, no. 23, pp. 11520-11524, 2005.

[33] H. Xie, M. Choi, and X. Zhang, "Self-organized carbon nanotube pellet and its thermal transport properties," in Proceedings of the 13th International Heat Transfer Conference (THP '06), Sydney, Australia, August 2006.

[34] G. Maurin, I. Stepanek, P. Bernier, J.-F. Colomer, J. B. Nagy, and F. Henn, "Segmented and opened multi-walled carbon nanotubes," Carbon, vol. 39, no. 8, pp. 1273-1278, 2001.

[35] D. A. Heller, P. W. Barone, J. P. Swanson, R. M. Mayrhofer, and M. S. Strano, "Using Raman spectroscopy to elucidate the aggregation state of single-walled carbon nanotubes," Journal of Physical Chemistry B, vol. 108, no. 22, pp. 6905-6909, 2004. 

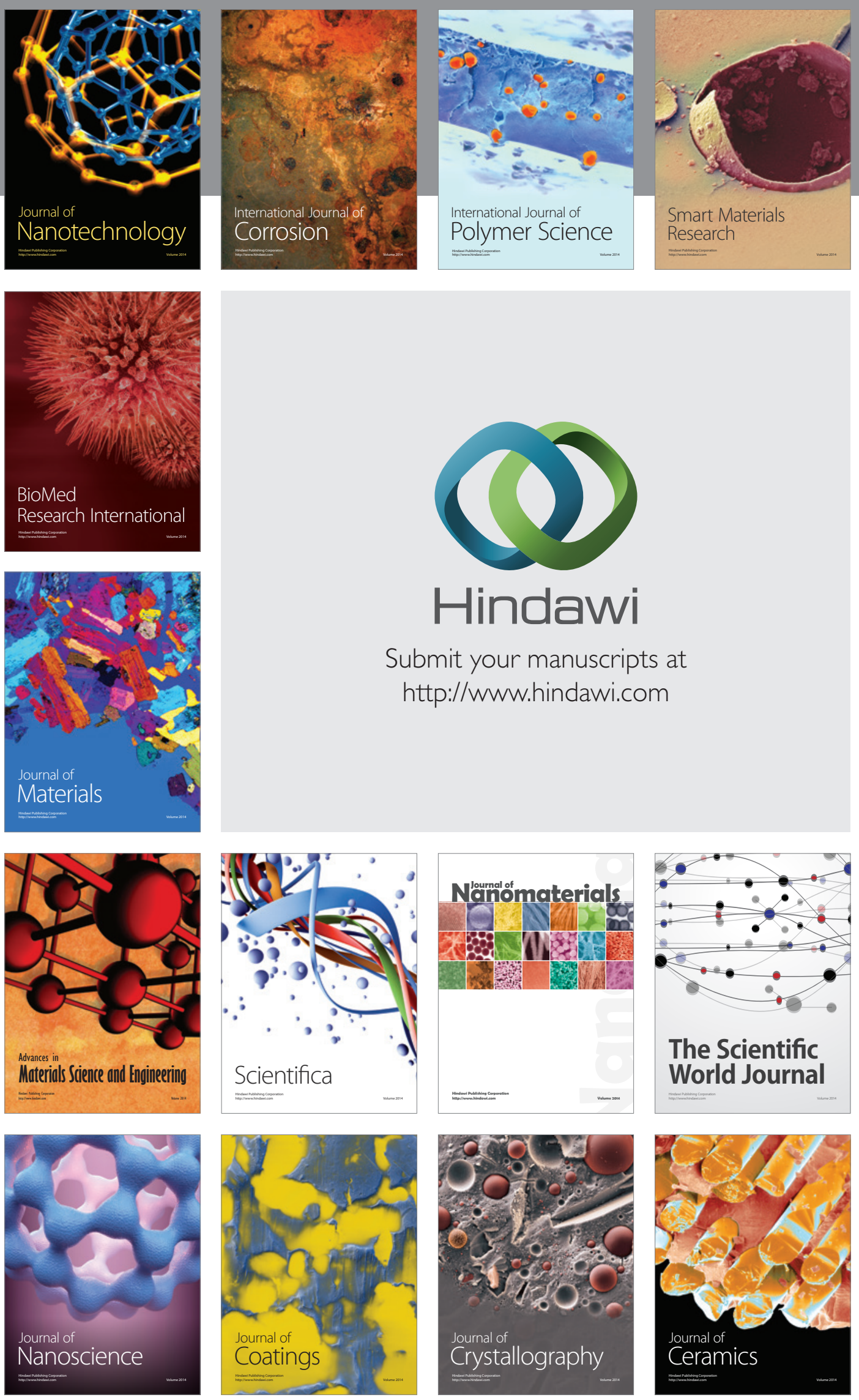

The Scientific World Journal

Submit your manuscripts at

http://www.hindawi.com

\section{World Journal}

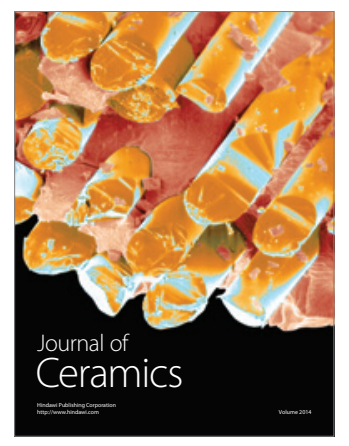

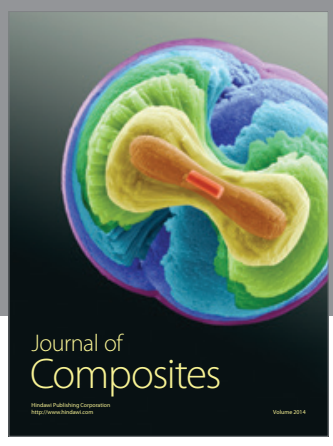
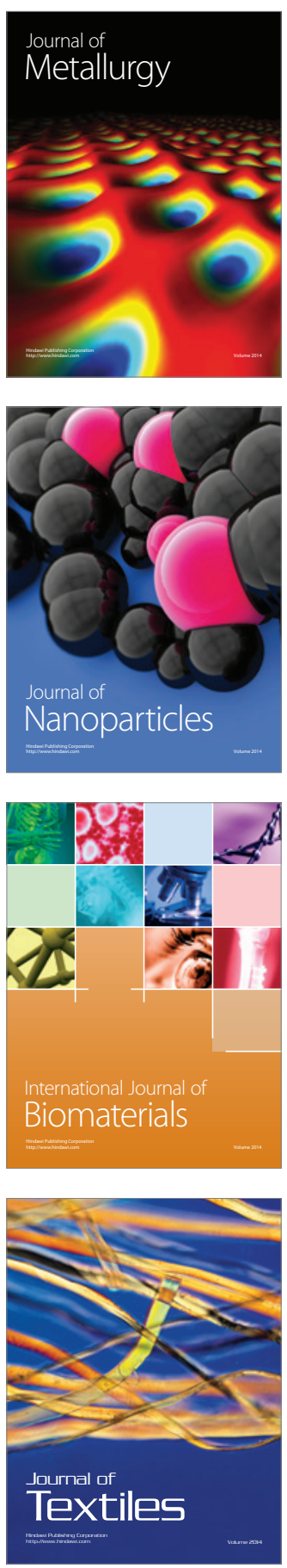